\title{
Kronecker representation and decompositional analysis of closed queueing networks with phase-type service distributions and arbitrary buffer sizes
}

\author{
Tuğrul Dayar · Akın Meriç
}

Published online: 13 September 2008

(C) Springer Science+Business Media, LLC 2008

\begin{abstract}
Two approximative fixed-point iterative methods based on decomposition for closed queueing networks with Coxian service distributions and arbitrary buffer sizes are extended to include phase-type service distributions. The irreducible Markov chain associated with each subnetwork in the respective decompositions is represented hierarchically using Kronecker products. The two methods are implemented in a software tool capable of computing the steady-state probability vector of each subnetwork by a multilevel method at each fixed-point iteration and are compared with other methods for accuracy and efficiency. Numerical results indicate that there is a niche filled by the two approximative methods.
\end{abstract}

Keywords Closed queueing network · Phase-type service distribution · Kronecker representation · Network decomposition · Fixed-point iteration $\cdot$ Multilevel method

Today, obtaining various performance measures for queueing networks (QNs) exactly (up to computer precision) still remains a challenging problem. Indeed, only a small class of QNs can be solved analytically (and exactly) for their performance measures (see, for instance, Baskett et al. 1975; Gordon and Newell 1967). This class of networks is called product form and requires specific conditions on the arrival processes, service processes, service disciplines, and buffer sizes of queues. On the other hand, obtaining exact performance measures for networks of queues with general arrival and service time distributions and arbitrary buffer sizes is not straightforward. The class of closed QNs with phase-type service distributions, first-come first-served (FCFS) service disciplines, and finite buffer sizes considered in this paper are among this latter kind of networks, since they are not product form and their state spaces grow exponentially with numbers of customers, queues, and phases in each queue. Furthermore, customers in these QNs may be subject to blocking because of the finite buffers

The authors thank Jean-Michel Fourneau for pointing out Marie's method and Brouwer's fixed-point theorem. The first author gratefully acknowledges grant TÜBA-GEBIP from the Turkish Academy of Sciences.

T. Dayar $(\bowtie) \cdot$ A. Meriç

Department of Computer Engineering, Bilkent University, 06800 Bilkent, Ankara, Turkey

e-mail: tugrul@cs.bilkent.edu.tr 
of some queues. In this paper, we assume that the customer subject to blocking is not lost, but forced to remain in the server until its destination queue's buffer is available to accept the customer. Hence, the number of customers in the class of closed QNs considered remains constant.

For steady-state analysis, one needs to solve the system of linear equations

$$
\pi Q=0, \quad \sum_{i \in \mathcal{S}} \pi_{i}=1,
$$

where $Q$ denotes the infinitesimal generator matrix of the irreducible Markov chain (MC) describing exponential transition rates among states of the particular closed QN, $\mathcal{S}$ is the set of states of $Q$, and $\pi$ is its steady-state probability (row) vector. When the infinitesimal generator matrix $Q$ is irreducible, then $\pi$ in (1) exists, is unique, and is positive (Stewart 1994, Chap. 1). By using Kronecker (or tensor) products (see, for instance, (Davio 1981)) of smaller matrices to represent $Q$ as in Buchholz (1994) and by performing vector-Kronecker product multiplications (see, for instance, Fernandes et al. 1998) within a multilevel (ML) iterative method as in Buchholz and Dayar (2004), it is possible to obtain $\pi$ without generating $Q$. This state-of-the-art approach results in important storage savings compared to sparse MC solvers and is generally considered to be the fastest solver for Kronecker structured MCs as discussed by Buchholz and Dayar (2007). For a recent review on analyzing MCs based on Kronecker products, see, for instance, Dayar (2006).

Several approximative methods for analyzing the steady-state behavior of closed QNs with arbitrary buffer sizes have been proposed. These methods are based on decomposing the QN into a set of subnetworks which satisfy certain properties. These subnetworks are analyzed in isolation to obtain marginal (or conditional) performance measures. This approach can be very efficient when the isolated subnetworks are simple to analyze and weakly coupled. Some methods force almost exact aggregation for closed QNs with arbitrary buffer sizes as in Altiok (1989), Frein and Dallery (1989), Marie (1979), Perros et al. (1988), Yao and Buzacott (1986). Some methods can be applied to networks with exponentially distributed service times as in Akyildiz (1988a), Frein and Dallery (1989), Perros et al. (1988), Suri and Diehl (1986), while others can be applied to those with phase-type service distributions as in Altiok (1989) or to those with Coxian service distributions as in Marie (1979), Yao and Buzacott (1986). The decomposition procedure introduces the first level of error while computing various performance measures for closed QNs with phase-type service distributions and arbitrary buffer sizes. QNs with phase-type service distributions can also be analyzed by methods that assume exponential service distributions. Yet, this introduces another level of error, because mean service rates of phase-type service distributions are used as if they were exponential service rates. In this respect, approximative methods for QNs with phase-type service distributions and arbitrary buffer sizes introduce less error and are more suitable for obtaining various performance measures.

It is shown by Gordon and Newell (1967) that closed QNs with exponential service distributions and infinite buffer sizes have product form solution. Thus, the steady-state distribution of such networks can be computed analytically using normalization constants exactly. Buzen (1973) devised a method known as the convolution algorithm to efficiently compute the normalization constants. Although the convolution algorithm can be used as an approximative method for computing performance measures of closed QNs with blocking, there are various methods proposed in the literature specifically for closed QNs with blocking and the ones considered in this paper are briefly reviewed next.

Marie (1979) proposed an approximation algorithm based on network decomposition to obtain the marginal steady-state distributions of a closed QN with Coxian service distributions and arbitrary buffer sizes. Marie's method decomposes the closed QN into a collection 
of subnetworks, where the transition probabilities between subnetworks are independent of the states of the subnetworks. Thus, each subnetwork is considered as an exponential service station with load dependent service rate for which the parameters of the equivalent server are obtained by analyzing the subnetwork in isolation under state dependent Poisson arrivals. Then the approximate results are obtained via a fixed-point iteration scheme. Numerical results show that the method provides a maximum relative error of $1 \%$ for throughput values and a maximum relative error of $7 \%$ for mean queue length values. Although Marie's method yields highly accurate results, a drawback of the method is that it analyzes the subnetworks numerically in a sparse setting which can be time consuming for large networks.

Yao and Buzacott (1986) proposed an approximation algorithm for closed QNs with Coxian service distributions and arbitrary buffer sizes. Their method decomposes the network into individual queues and approximates the service distribution of each queue by an exponential distribution with the same mean as that of the original Coxian server. Experimental results provide a maximum relative error of $2 \%$ for throughput values. It is remarked that the method should be mostly adequate when applied to closed QNs with a moderate number of queues and customers.

Akyildiz (1988a) developed an algorithm for approximating the throughput of closed QNs with exponential service distributions. The idea behind his approximation algorithm is that the throughput of a blocking closed QN is approximately the same as an equivalent nonblocking closed QN which has product form queue length distribution. In that respect, the number of customers of the equivalent closed QN without blocking is chosen such that the number of states of the closed QN with blocking is close to the number of states of the closed QN without blocking. The QN under consideration is assumed to be deadlock free, and if blocking occurs, then customers will face blocking after service. Akyildiz's method can produce throughput values with relative error smaller than $2 \%$ for closed QNs with blocking and exponential service distributions. Yet, it is unable to produce accurate results for other performance measures or for networks with phase-type service distributions. Akyildiz (1988b) also proposed mean value analysis for closed QNs with blocking after service. The proposed method is based on the arrival theorem and extends the classical mean value analysis of Reiser and Lavenberg (1980) to include finite queues.

In this paper, we extend two approximative fixed-point iterative methods based on decomposition for closed QNs with Coxian service distributions and arbitrary buffer sizes from the literature to include phase-type service distributions. These are Marie's (M) method and Yao and Buzacott's (YB) method. We show how the irreducible MC associated with each subnetwork in the respective decompositions can be represented hierarchically using Kronecker products. The decompositional nature of the methods imply an additive dimension of scalability. The Kronecker representation of each subnetwork model in the decomposition facilitates yet another form of compactness and a multiplicative dimension of scalability. Since, the methods are already approximative by construction, the closed QN model becomes essentially more compact with the Kronecker representation.

The proposed methods are implemented in a software tool by Meriç (2007b) such that the steady-state vector of each subnetwork is computed by the ML method at each fixedpoint iteration. The methods of M and YB are compared with the ML and successive overrelaxation (SOR) (see Uysal and Dayar 1998) methods for the closed QN itself and with the convolution algorithm (CA) of Buzen (1973) and mean value analysis (MVABLO) of Akyildiz (1988b), for accuracy and efficiency on a number of examples using the tool. The reason behind using CA and MVABLO is that these methods are approximative analytical methods unlike the methods of $\mathrm{M}$ and $\mathrm{YB}$ and need almost no computational effort. Hence, this comparison may reveal when it is worthwhile to use the approximative iterative methods 
of $\mathrm{M}$ and $\mathrm{YB}$. SOR is included in order to make a comparison with the ML method. In this way, we intend to fill a niche accuracywise and efficiencywise between iterative methods that can be executed until a tolerance close to computer precision and approximative analytical methods whose accuracy cannot be forecasted beforehand.

In Sect. 1, we provide a Kronecker representation for the class of closed QNs considered and briefly explain the ML method. In Sect. 2, we discuss the methods of M and YB. Therein, it is shown how the subnetworks obtained by the decomposition of the closed QN model in the methods of M and YB can be represented using Kronecker products. In Sect. 3, we present the results of numerical experiments, and in Sect. 4, we conclude.

\section{Kronecker representation and ML method}

In this section, we provide a brief overview of Kronecker algebra and give a formal definition of the closed QN model used. A small example is also included in order to clarify the discussion. Then we introduce the ML method used in solving MCs expressed in terms of Kronecker products.

Throughout the text, we denote matrices by upper-case letters, a block of a matrix by specifying the indices of the block in parentheses beside the matrix name, and an element of a matrix by specifying the indices of the element as subscripts of the lower-case matrix name. Since we model closed QNs and queues can be empty, it is natural to let rows and columns of matrices representing evolution of queues be numbered starting from zero. We recall that the Kronecker product of two (rectangular) matrices $A \in \mathbb{R}^{r_{A} \times c_{A}}$ and $B \in \mathbb{R}^{r_{B} \times c_{B}}$ is written as $A \otimes B$ and yields the matrix $X \in \mathbb{R}^{r_{A} r_{B} \times c_{A} c_{B}}$, whose elements satisfy $x_{i_{A} r_{B}+i_{B}, j_{A} c_{B}+j_{B}}=a_{i_{A}, j_{A}} b_{i_{B}, j_{B}}$, and $\times$ denotes the Cartesian product operator. The Kronecker sum of two square matrices $F \in \mathbb{R}^{r_{F} \times r_{F}}$ and $G \in \mathbb{R}^{r_{G} \times r_{G}}$ is written as $F \oplus G$ and yields the matrix $Y \in \mathbb{R}^{r_{F} r_{G} \times r_{F} r_{G}}$, which is defined in terms of two Kronecker products as $Y=F \otimes I_{r_{G}}+I_{r_{F}} \otimes G$. Here $I_{r_{F}}$ and $I_{r_{G}}$ denote identity matrices of orders $r_{F}$ and $r_{G}$, respectively. The Kronecker product and Kronecker sum are associative and defined for more than two matrices (see, for instance, Davio 1981).

\subsection{Kronecker representation}

We consider a class of closed FCFS QNs with arbitrary buffer sizes and phase-type service distributions defined by $J$ queues, $K$ customers, routing probability matrix $P$, phase-type distribution $\left(\alpha^{(j)}, T^{(j)}\right)$, where $T^{(j)}$ is the phase-type distribution matrix of order $t_{j}$ and $\alpha^{(j)}$ is the initial probability distribution row vector of length $t_{j}$ associated with $T^{(j)}$, and buffer size $b_{j}$ for queue $j \in\{1,2, \ldots, J\}$. We let $c_{j}=\min \left\{K, b_{j}\right\}$ and the state of queue $j$ be represented by the ordered pair $i_{j}=\left(n_{j}, \phi_{j}\right)$, where $n_{j} \in\left\{0,1, \ldots, c_{j}\right\}$ denotes the occupancy of queue $j$ and $\phi_{j} \in\left\{0,1, \ldots, t_{j}-1\right\}$ denotes the phase of its service process, with the constraint that $\phi_{j}=0$ when $n_{j}=0$ (that is, phase is irrelevant when the queue is empty). Then $i_{j} \in\{(0,0)\} \cup\left\{1,2, \ldots, c_{j}\right\} \times\left\{0,1, \ldots, t_{j}-1\right\}$. We remark that in our model, an arrival to a destination queue can only take place when the destination queue has space for the arriving customer; otherwise the transition is inhibited. The implication of this assumption is that a customer will remain in the server until space becomes available in the destination queue.

The irreducible MC representing the evolution of queue $j \in\{1,2, \ldots, J\}$ is a $\left(c_{j}+1\right) \times$ $\left(c_{j}+1\right)$ block tridiagonal matrix and is given by $Q^{(j)}=G^{(j)}+D^{(j)}$ (see, for instance, 
Buchholz 1994), where

$$
G^{(j)}=\left(\begin{array}{cccc}
O^{(j)}(0,0) & \lambda_{j}(0) A^{(j)}(0,1) & & \\
S^{(j)}(1,0) & O^{(j)}(1,1) & \lambda_{j}(1) A^{(j)}(1,2) & \\
\ddots & \ddots & \ddots & \lambda_{j}\left(c_{j}-1\right) A^{(j)}\left(c_{j}-1, c_{j}\right) \\
& S^{(j)}\left(c_{j}-1, c_{j}-2\right) & O^{(j)}\left(c_{j}-1, c_{j}-1\right) & O^{(j)}\left(c_{j}, c_{j}\right)
\end{array}\right),
$$

$\bar{T}^{(j)}=-T^{(j)} e, e$ is the column vector of ones of appropriate length, $O^{(j)}\left(n_{j}, n_{j}\right)=T^{(j)}$ for $n_{j} \in\left\{1,2, \ldots, c_{j}\right\}, O^{(j)}(0,0)=0, S^{(j)}\left(n_{j}, n_{j}-1\right)=\bar{T}^{(j)} \alpha^{(j)}$ for $n_{j} \in\left\{2,3, \ldots, c_{j}\right\}$, $S^{(j)}(1,0)=\bar{T}^{(j)}, A^{(j)}\left(n_{j}, n_{j}+1\right)=I_{t_{j}}$ for $n_{j} \in\left\{1,2, \ldots, c_{j}-1\right\}, A^{(j)}(0,1)=\alpha^{(j)}, \lambda_{j}\left(n_{j}\right)$ is the rate of arrivals to queue $j$ under buffer occupancy $n_{j}$, and $D^{(j)}$ is the diagonal correction matrix summing the rows of $Q^{(j)}$ to zero. The upper-diagonal blocks $G^{(j)}\left(n_{j}, n_{j}+1\right)$ and lower-diagonal blocks $G^{(j)}\left(n_{j}, n_{j}-1\right)$ of $G^{(j)}$ represent the arrivals of customers and service completions, respectively. Its diagonal blocks $G^{(j)}\left(n_{j}, n_{j}\right)$ represent phase changes. The boundary level has a single state, while the other levels each have $t_{j}$ states. Hence, $G^{(j)}$ is a $\left(t_{j} c_{j}+1\right) \times\left(t_{j} c_{j}+1\right)$ matrix.

Assuming that the states of the irreducible MC underlying the closed QN are represented as $i=\left(i_{1}, i_{2}, \ldots, i_{J}\right)$, let us define the mapping $f: \mathcal{S} \rightarrow \mathcal{N}$ as

$$
f(i)=f\left(\left(i_{1}, i_{2}, \ldots, i_{J}\right)\right)=f\left(\left(\left(n_{1}, \phi_{1}\right),\left(n_{2}, \phi_{2}\right), \ldots,\left(n_{J}, \phi_{J}\right)\right)\right)=\left(n_{1}, n_{2}, \ldots, n_{J}\right)=n
$$

for $i \in \mathcal{S}$ (see (1)) and $n=\left(n_{1}, n_{2}, \ldots, n_{J}\right) \in \mathcal{N}$. This mapping is onto and partitions $\mathcal{S}$ into equivalence classes. The set of equivalence classes defined by $f$ is denoted as $\mathcal{N}$ and has cardinality $|\mathcal{N}|$. We remark that $n \in \mathcal{N}$ is represented using the row vector $\left(n_{1}, n_{2}, \ldots, n_{J}\right)$, which satisfies $\sum_{j=1}^{J} n_{j}=K$.

When queues are interconnected to form a closed QN, the arrival rate of customers to queue $j \in\{1,2, \ldots, J\}$, that is, $\lambda_{j}\left(n_{j}\right)$, depends on column $j$ of the routing probability matrix, $P$, the states of the queues corresponding to nonzero elements in that column, and the rates by which the queues complete the last phases of their service processes. Assuming the lexicographical order is associated with the states in $\mathcal{N}$, the generator matrix $Q$ of a closed QN can be expressed as an $(|\mathcal{N}| \times|\mathcal{N}|)$ block matrix with block $(n, m)$ for $n, m \in \mathcal{N}$ given in (Buchholz 1994, p. 66) by

$$
Q(n, m)= \begin{cases}Q^{\{j, k\}}(n, m), & n \neq m \text { and } m=n-e_{j}^{T}+e_{k}^{T} \\ D(n, n)+Q(n, n)_{D}+\sum_{j=1}^{J} Q^{\{j, j\}}(n, n), & n=m \\ 0 & \text { otherwise }\end{cases}
$$

where $j, k \in\{1,2, \ldots, J\}, e_{j}$ is column $j$ of the identity matrix, $m=n-e_{j}^{T}+e_{k}^{T}$ refers to service completion at queue $j$ and arrival to queue $k$ when in state $n$ so as to make a transition to state $m, D(n, n)$ is the diagonal matrix summing block $n$ of rows in $Q$ to zero,

$$
Q^{\{j, k\}}(n, m)= \begin{cases}p_{j, k}\left(I_{c_{j}^{\{j, k\}}} \otimes S^{(j)}\left(n_{j}, m_{j}\right) \otimes I_{r_{j}^{\{j, k\}}}\right)\left(I_{c_{k}^{\{j, k\}}} \otimes A^{(k)}\left(n_{k}, m_{k}\right) \otimes I_{r_{k}^{\{j, k\}}}\right), & j<k \\ p_{j, k}\left(I_{c_{k}^{\{j, k\}}} \otimes A^{(k)}\left(n_{k}, m_{k}\right) \otimes I_{r_{k}^{\{j, k\}}}\right)\left(I_{c_{j}^{\{j, k\}}} \otimes S^{(j)}\left(n_{j}, m_{j}\right) \otimes I_{\left.r_{j}^{\{j, k\}}\right),}\right. & j>k, \\ p_{j, j}\left(I_{c_{j}^{\{j, j\}}} \otimes S^{(j)}\left(n_{j}, n_{j}-1\right)\right)\left(A^{(j)}\left(n_{j}, n_{j}+1\right) \otimes I_{r_{j}^{\{j, j\}}}\right), & j=k\end{cases}
$$


Fig. 1 A closed QN

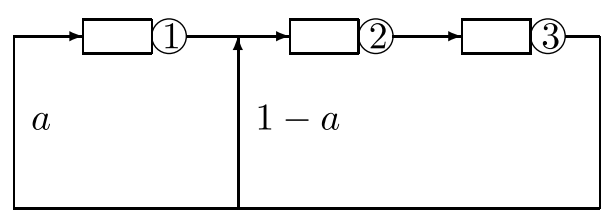

$$
Q(n, n)_{D}=\bigoplus_{j=1}^{J} O^{(j)}\left(n_{j}, n_{j}\right)=\sum_{j=1}^{J} I_{c_{j}^{\{j, j\}}} \otimes O^{(j)}\left(n_{j}, n_{j}\right) \otimes I_{r_{j}^{\{j, j\}}},
$$

$c_{x}^{\{j, k\}}=\prod_{z=1}^{x-1} \operatorname{siz} e^{\{j, k\}}(z)$ and $r_{x}^{\{j, k\}}=\prod_{z=x+1}^{J} \operatorname{siz} e^{\{j, k\}}(z)$ represent products of column and row dimensions of matrices respectively, and

$$
\operatorname{size} e^{\{j, k\}}(z)=\left\{\begin{array}{ll}
\text { \#_of_rows }\left(O^{(z)}\left(n_{z}, n_{z}\right)\right), & z \neq j \text { and } z \neq k \\
\text { \#_of_rows }\left(A^{(k)}\left(n_{k}, m_{k}\right)\right), & z=k \text { and } k>j \\
\text { \#_of_cols }\left(A^{(k)}\left(n_{k}, m_{k}\right)\right), & z=k \text { and } k<j \\
\text { \#_of_rows }\left(S^{(j)}\left(n_{j}, m_{j}\right)\right), & z=j \text { an d } j>k \\
\text { \#_of_cols }\left(S^{(j)}\left(n_{j}, m_{j}\right)\right), & z=j \text { and } j<k
\end{array} .\right.
$$

\section{Example 1}

Consider the closed QN in Fig. 1, which consists of three queues, two customers, and the routing probability matrix

$$
P=\frac{1}{2}\left(\begin{array}{ccc}
1 & 2 & 3 \\
3 & 1 & 0 \\
0 & 0 & 1 \\
a & 1-a & 0
\end{array}\right)
$$

with $0<a<1$. Hence, $N=3$ and $K=2$, the service distributions and buffer sizes of queues are given by

$$
\begin{aligned}
& T^{(1)}=\left(-\mu_{1}^{(1)}\right), \quad \alpha^{(1)}=(1), \quad b_{1}=2, \\
& T^{(2)}=\left(\begin{array}{cc}
-\mu_{1}^{(2)} & \mu_{1}^{(2)} \\
0 & -\mu_{2}^{(2)}
\end{array}\right), \quad \alpha^{(2)}=(1,0), \quad b_{2}=2, \\
& T^{(3)}=\left(\begin{array}{cc}
-\mu_{1}^{(3)} & \mu_{1}^{(3)} \\
0 & -\mu_{2}^{(3)}
\end{array}\right), \quad \alpha^{(3)}=(1,0), \quad b_{3}=1 .
\end{aligned}
$$

Queue 1 has an exponential service distribution, queues 2 and 3 have hypoexponential service distributions with capacities $c_{1}=c_{2}=2$ and $c_{3}=1$.

For this example, $\mathcal{N}$ is obtained from $\mathcal{S}$ using (2) as in Table 1 . The state space $S$ consists of 11 states, which are divided into 5 equivalence classes with cardinalities $4,2,2,2,1$. Observe that $p_{1,1}=p_{2,2}=p_{3,3}=0$. This implies that the third (summation) term in (2) for $n=m$ evaluates to zero, since $Q^{\{j, j\}}(n, n)=0$ for $j \in\{1,2,3\}$ and $n \in \mathcal{N}$. For illustrative purposes, we show how blocks $((0,2,0),(0,2,0))$ and $((0,1,1),(0,2,0))$ of $Q$ are computed from (2); we represent diagonal elements of $Q$ using *'s. 
Table 1 State space $\mathcal{S}$ versus set of equivalence classes $\mathcal{N}$ for Example 1

\begin{tabular}{cr}
$\mathcal{S}$ & $\mathcal{N}$ \\
\hline$((\underline{0}, 0),(\underline{1}, 1),(\underline{1}, 1)),((\underline{0}, 0),(\underline{1}, 1),(\underline{1}, 2)),((\underline{0}, 0),(\underline{1}, 2),(\underline{1}, 1)),((\underline{0}, 0),(\underline{1}, 2),(\underline{1}, 2))$ & $(0,1,1)$ \\
$((\underline{0}, 0),(\underline{2}, 1),(\underline{0}, 0)),((\underline{0}, 0),(\underline{2}, 2),(\underline{0}, 0))$ & $(0,2,0)$ \\
$((\underline{1}, 1),(\underline{0}, 0),(\underline{1}, 1)),((\underline{1}, 1),(\underline{0}, 0),(\underline{1}, 2))$ & $(1,0,1)$ \\
$((\underline{1}, 1),(\underline{1}, 1),(\underline{0}, 0)),((\underline{1}, 1),(\underline{1}, 2),(\underline{0}, 0))$ & $(1,1,0)$ \\
$((\underline{2}, 1),(\underline{0}, 0),(\underline{0}, 0))$ & $(2,0,0)$ \\
\hline
\end{tabular}

$$
\begin{aligned}
Q((0,2,0),(0,2,0))= & D((0,2,0),(0,2,0))+Q((0,2,0),(0,2,0))_{D} \\
& +\sum_{j=1}^{3} Q^{\{j, j\}}((0,2,0),(0,2,0)) \\
= & D((0,2,0),(0,2,0))+I_{c_{1}^{\{1,1\}}} \otimes O^{(1)}(0,0) \otimes I_{r_{1}^{\{1,1\}}} \\
& +I_{c_{2}^{\{2,2\}}} \otimes O^{(2)}(2,2) \otimes I_{r_{2}^{\{2,2\}}}+I_{c_{3}^{\{3,3\}}} \otimes O^{(3)}(0,0) \otimes I_{r_{3}^{\{3,3\}}} \\
= & D((0,2,0),(0,2,0))+(0) \otimes I_{2} \\
& +I_{1} \otimes\left(\begin{array}{cc}
-\mu_{1}^{(2)} & \mu_{1}^{(2)} \\
0 & -\mu_{2}^{(2)}
\end{array}\right) \otimes I_{1}+I_{2} \otimes(0)=\left(\begin{array}{cc}
* & \mu_{1}^{(2)} \\
0 & *
\end{array}\right) .
\end{aligned}
$$

$$
\begin{aligned}
Q((0,1,1),(0,2,0)) & =Q^{\{3,2\}}((0,1,1),(0,2,0)) \\
& =p_{3,2}\left(I_{c_{2}^{\{3,2\}}} \otimes A^{(2)}(1,2) \otimes I_{r_{2}^{\{3,2\}}}\right)\left(I_{c_{3}^{\{3,2\}}} \otimes S^{(3)}(1,0) \otimes I_{r_{3}^{\{3,2\}}}\right) \\
& =p_{3,2}\left(I_{1} \otimes\left(\begin{array}{cc}
1 & 0 \\
0 & 1
\end{array}\right) \otimes I_{2}\right)\left(I_{2} \otimes\left(\begin{array}{c}
0 \\
\mu_{2}^{(3)}
\end{array}\right)\right) \\
& =(1-a)\left(I_{4}\right)\left(\begin{array}{cc}
0 & 0 \\
\mu_{2}^{(3)} & 0 \\
0 & 0 \\
0 & \mu_{2}^{(3)}
\end{array}\right)=\left(\begin{array}{cc}
(1-a) \mu_{2}^{(3)} & 0 \\
0 & 0 \\
0 & (1-a) \mu_{2}^{(3)}
\end{array}\right) .
\end{aligned}
$$

Thus, we have the generator matrix

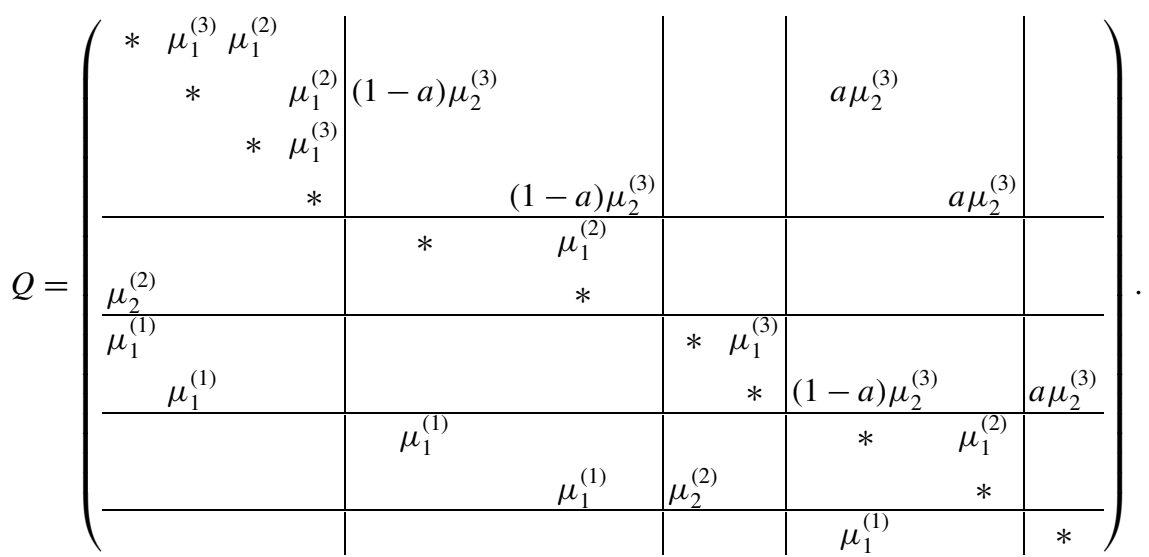


Hierarchical representation of QNs consists of a multilevel structure and assumes information abstraction between levels Buchholz (1994). The highest level structure, called high level model (HLM), determines the routing among lower level structures, called low level models (LLMs). LLMs, either consist of queues or are themselves structures. The HLM defines the routing between lower level structures. Any piece of information belonging to a particular level is hidden from subsequent levels. In our representation, we restrict closed QN models to have queues as LLMs and define the interaction among LLMs by an HLM.

In order to define the HLM, we need to specify the transitions among LLMs. There are two ingredients that help us to reveal these transitions: one is the set $\mathcal{N}$ and the other is the routing probability matrix $P$. Since each component of $n \in \mathcal{N}$ corresponds to the number of customers in an LLM, possible transitions from $n$ to $m$, where $n, m \in \mathcal{N}$, can be determined by considering $P$ and $m=n-e_{j}^{T}+e_{k}^{T}$ as discussed after (2). These transitions are represented by an $(|\mathcal{N}| \times|\mathcal{N}|)$ matrix called the HLM matrix; elements of $\mathcal{N}$ are named as states of the HLM.

Example 1 consists of 3 LLMs and 5 HLM states, resulting with a generator matrix, $Q$, of order 11. There are 9 transitions among HLM states, corresponding to blocks $((0,1,1),(0,2,0)),((0,1,1),(1,1,0)),((0,2,0),(0,1,1)),((1,0,1),(0,1,1)),((1,0,1),(1,1,0))$, $((1,0,1),(2,0,0)),((1,1,0),(0,2,0)),((1,1,0),(1,0,1))$, and $((2,0,0),(1,1,0))$ of $Q$ obtained by using Kronecker products and 5 local transitions corresponding to diagonal blocks of $Q$ obtained by using Kronecker sums. Thus, the HLM matrix of order 5 has 14 nonzeros with local transitions along the diagonal and transitions that result from movement of customers between queues in the off-diagonal. Each nonzero element in the HLM matrix corresponds to a Kronecker product of 3 LLM matrices, which are defined by the arrival and service processes of queues.

In practice, $Q$ is neither generated nor stored; instead an efficient vector-Kronecker product multiplication algorithm is used to carry out the steady-state analysis of $Q$ underlying the closed QN. A state-of-the-art method that can be used towards this end is introduced in the next section.

\subsection{ML method}

To obtain the steady-state vector of the generator matrix of a closed QN which is hierarchically modeled as described, an ML method originating from aggregation-disaggregation and using multigrid iteration as in Buchholz and Dayar (2004) can be employed. The ML method, which is capable of aggregating LLMs according to a fixed or circular order using V, W, or F cycles, is given in Algorithms 1 and 2 in (Buchholz and Dayar 2007, pp. 10321033). Let $l \in\{0,1, \ldots, J\}$ define the current level in the hierarchy. Then one $\mathrm{V}$ cycle of the ML method proceeds as follows. At the finest level, $l=0$, a number of iterations (or smoothings) with one of the iterative methods Power, Jacobi over-relaxation (JOR), or SOR are applied to the vector $x^{(0)}$ with uniformly distributed elements using a splitting of the generator matrix $Q_{0}=Q$ as discussed in Uysal and Dayar (1998) and a smoothed vector $\tilde{x}^{(0)}$ is obtained. Then, for the next level, $l=1, Q_{0}$ is aggregated with respect to an LLM by using the smoothed vector $\tilde{x}^{(0)}$. Thus the elements of $\tilde{x}^{(0)}$ and the blocks of $Q_{0}$, which correspond to elements of the HLM matrix, are aggregated to obtain $x^{(1)}$ and $Q_{1}$, respectively. In the $(l+1)$ st aggregation step, $l<J$, the smoothed vector $\tilde{x}^{(l)}$ and the matrix $Q_{l}$ are used in the aggregation procedure to obtain $x^{(l+1)}$ and $Q_{l+1}$. At the coarsest level, where all LLMs become aggregated, $Q$ collapses to the aggregated matrix $Q_{J}$ of order $|\mathcal{N}|$, and the linear system

$$
x^{(J)} Q_{J}=0, \quad \sum_{n \in \mathcal{N}} x_{n}^{(J)}=1
$$


is solved exactly. At this point, the ML method starts to move in the reverse direction, from the coarsest level to the finest, performing a number of iterations at each level after disaggregation. In this way, the solution vector $x^{(J)}$ at the coarsest level is mapped back to the finest level. At the finest level, when a cycle finishes, the method computes the residual vector and either stops if a predefined tolerance is met or proceeds for another cycle.

In the next section, we provide a framework for two approximative decompositional methods to utilize the ML method in analyzing subnetworks.

\section{Two approximative decompositional methods}

In this section, we extend Marie's and Yao and Buzacott's methods to include phase-type service distributions and discuss how the subnetworks in the respective decompositions can be modeled hierarchically using Kronecker products. The existence of fixed-points for Marie's and Yao and Buzacott's methods is shown in (Meriç 2007a, Chap. 4) by arguing that both fixed-point systems of equations satisfy the conditions of Brouwer's fixed-point theorem as in Mainkar and Trivedi (1996). Space and time complexity analyses of the two methods and the CA, MVABLO, SOR, and ML methods are also given in (Meriç 2007a, Chap. 4). We refrain from including these results here due to space limitations.

\subsection{Marie's method}

Marie's method consists of two stages. In the first stage (i.e., step 1 in Table 2), it decomposes the closed QN into subnetworks, and in the second stage it sets the throughputs of queues to some initial values (i.e., step 2 in Table 2) and improves the throughputs of subnetworks using a fixed-point iteration scheme (i.e., steps 3 through 5 in Table 2). The decomposition satisfies some specific conditions, which assume that the steady-state probabilities of a subnetwork are independent of the states of other subnetworks. Thus, in the fixed-point iteration scheme, these independent subnetworks are analyzed in isolation as open QNs assuming they have state dependent Poisson arrival rates.

Table 2 Marie's method versus Yao and Buzacott's method

\begin{tabular}{ll}
\hline Marie's method & Yao and Buzacott's method \\
\hline Step 1. Decompose the closed QN into subnetworks. & $\begin{array}{l}\text { Step 1. Set state dependent exponential ser- } \\
\text { vice rates of queues } \mu \text { to some initial values. }\end{array}$ \\
$\begin{array}{l}\text { Step 2. Set throughput values of subnetworks } v \text { to } \\
\text { some initial values. }\end{array}$ & $\begin{array}{l}\text { Shich consists of queues with state depen- } \\
\text { dent exponential service rates, and obtain } \\
\text { steady-state probabilities } \pi \text { exp }\end{array}$
\end{tabular}

Step 3. Compute state dependent arrival rates $\lambda$ for each subnetwork.

Step 4. Analyze subnetworks as open QNs under state dependent Poisson arrival rates $\lambda$ to derive steadystate probabilities $\pi$.

Step 5. Compute new throughput values $v$ using $\lambda$ and $\pi$, and goto Step 3 until convergence.
Step 3. Compute state dependent arrival rates $\lambda$ for each queue using $\pi^{\exp }$.

Step 4. Analyze each queue in isolation with its original service distribution and state dependent Poisson arrival rates $\lambda$ and derive steady-state probabilities $\pi$.

Step 5. Compute new throughput values $v$ using $\lambda$ and $\pi$, initialize $\mu$ with $\nu$ and goto Step 2 until convergence. 
In the first stage, the decomposition of queues into subnetworks depend on buffer sizes of queues and number of customers in the closed QN, and is described as follows. Given a closed QN, customers arriving to finite buffer queues of a subnetwork must come from queues that belong to the same subnetwork. In other words, any upstream queue whose departing customers are directed to a finite buffer queue must be in the same subnetwork with the finite buffer queue. The decomposition is computed recursively by Algorithms 6 and 7 in (Meriç 2007a, pp. 44-45). Using these algorithms, the set of queue indices $\mathcal{I}=$ $\{1,2, \ldots, J\}$ of the closed QN is partitioned into subsets $\mathcal{J}^{(k)}$ of $\mathcal{I}$, where $k \in\{1,2, \ldots, S\}$ and $S$ is the number of subnetworks. Hence, the closed QN in Example 1 is partitioned into the two subnetworks $\mathcal{J}^{(1)}=\{1\}$ and $\mathcal{J}^{(2)}=\{2,3\}$.

In the second stage, a fixed-point iteration scheme is employed to obtain approximations to throughputs of subnetworks. This scheme is defined by Marie (1979) using the system of equations

$$
\begin{aligned}
\alpha & =\left(\alpha_{1}, \alpha_{2}, \ldots, \alpha_{S}\right), A_{m}^{(r-1)}\left(K_{m}\right)=\left\{\begin{array}{l}
1 \\
\prod_{k=1}^{K_{m}} v_{m}^{(r-1)}(k) \text { if } \quad K_{m}>0
\end{array},\right. \\
N C_{j}^{(r-1)}(n) & =\sum_{K_{1}, K_{2}, \ldots, K_{S}}\left(\prod_{m=1}^{S} \frac{\alpha_{m}^{K_{m}}}{A_{m}^{(r-1)}\left(K_{m}\right)}\right) \text { and } \sum_{m=1}^{S} K_{m}=n, K_{j}=0, \\
\lambda_{j}^{(r-1)}(l) & =\frac{\alpha_{j} N C_{j}^{(r-1)}(K-l-1)}{N C_{j}^{(r-1)}(K-l)}, \quad l \in\{0,1, \ldots, K-1\}, \\
v_{j}^{(r)}(i) & =\frac{\alpha_{j} N C_{j}^{(r-1)}(K-i)}{N C_{j}^{(r-1)}(K-i+1)} \frac{\pi_{j}^{(r-1)}(i-1)}{\pi_{j}^{(r-1)}(i)}, \quad i \in\{1,2, \ldots, K\}, j \in\{1,2, \ldots, S\},
\end{aligned}
$$

where $\lambda_{j}^{(r)}(l)$ and $v_{j}^{(r)}(i)$ denote the Poisson arrival rate and the throughput of $\mathcal{J}^{(j)}$ when it has $l$ and $i$ customers at iteration $r$, respectively. Furthermore, $\pi_{j}^{(r)}$ is the steady-state vector of $\mathcal{J}^{(j)}, \pi_{j}^{(r)}(i)$ is the marginal steady-state probability of having $i$ customers in $\mathcal{J}^{(j)}$ at iteration $r$, and

$$
\alpha_{j}=\sum_{i \in \mathcal{J}^{(j)}} x_{i}\left(\sum_{k \in I / \mathcal{J}^{(j)}} p_{i, k}\right) \text { for } j \in\{1,2, \ldots, S\}
$$

defines the visit ratio of $\mathcal{J}^{(j)}$ for which $x$ is a solution of $x P=x$ subject to $x_{1}=1$. The arrival rate and steady-state distribution vectors for $\mathcal{J}^{(j)}$ at iteration $r$ are then given by $\lambda_{j}^{(r)}=\left(\lambda_{j}^{(r)}(0), \lambda_{j}^{(r)}(1), \ldots, \lambda_{j}^{(r)}(K-1)\right)$ and $\pi_{j}^{(r)}=\left(\pi_{j}^{(r)}(0), \pi_{j}^{(r)}(1), \ldots, \pi_{j}^{(r)}(K)\right)$, respectively. Observe that the computation of $\lambda_{j}^{(r-1)}$ and $\nu_{j}^{(r)}$ for $j \in\{1,2, \ldots, S\}$ can be vectorized without difficulty if the elementwise division operator for two vectors of the same length is available.

At iteration $r, \mathcal{J}^{(j)}$ is perceived as an open QN with state dependent Poisson arrival rates $\lambda_{j}^{(r)}$ and analyzed for its steady-state vector. In order to carry out the steady-state analysis, the open QN is modeled as a closed QN, which consists of the subnetwork's queues and a slack queue as in Fig. 2. The slack queue is an infinite buffer queue, simulates the state dependent Poisson arrivals of customers to the subnetwork, and has exponentially distributed service times with load dependent service rates. In this manner, subnetworks of Example 1, defined by $\mathcal{J}^{(1)}$ and $\mathcal{J}^{(2)}$, are modeled as in Fig. 3 .

Each closed QN in Fig. 3 is modeled hierarchically by defining the queues in the subnetwork and the slack queue as LLMs, and constructing the HLM matrix which represents the 
Fig. 2 Open QN modeled as closed QN with a slack queue
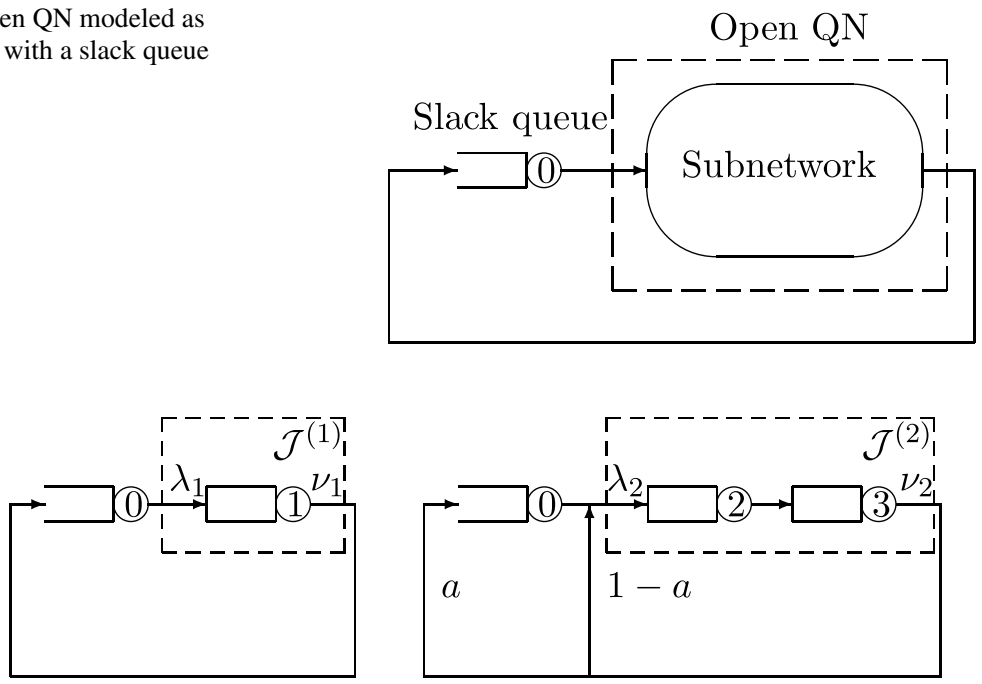

Fig. 3 Decomposition of Example 1 for Marie's method

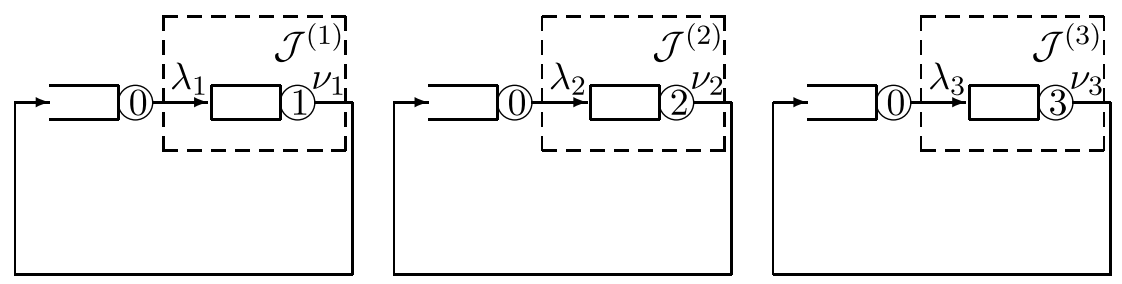

Fig. 4 Decomposition of Example 1 for Yao and Buzacott's method

interactions among the queues. Consequently, the ML method is utilized to compute $\pi_{j}^{(r)}$ for $j \in\{1,2, \ldots, S\}$ in step 4 of Marie's method in Table 2 at iteration $r$.

\subsection{Yao and Buzacott's method}

The idea behind Yao and Buzacott's method is to approximate a closed QN as an exponential network, where each queue has an exponentially distributed service time with state dependent rate. The decomposition in this approach is maximal in the sense that each queue is placed in a separate subnetwork. Hence, the closed QN in Example 1 is partitioned into the three subnetworks $\mathcal{J}^{(1)}=\{1\}, \mathcal{J}^{(2)}=\{2\}$, and $\mathcal{J}^{(3)}=\{3\}$. A slack queue with an infinite buffer and a state dependent exponential service rate is used to model state dependent Poisson arrivals with rate $\lambda_{j}$ to the queue in $\mathcal{J}^{(j)}$ having a phase-type service distribution for $j \in\{1,2, \ldots, J\}$ (see Fig. 4). After this approximation, the method sets the state dependent service rate, $\mu_{j}$, of the slack queue in $\mathcal{J}^{(j)}$ to some initial value for $j \in\{1,2, \ldots, J\}$ (i.e., step 1 in Table 2) and then employs a fixed-point iteration scheme on the decomposed network to improve the throughputs of queues (i.e., steps 2 through 5 in Table 2).

The fixed-point iteration of Yao and Buzacott (1986) is based on the system of equations

$$
\pi^{\exp ^{(r-1)}} Q^{\exp ^{(r-1)}}=0, \quad \sum_{n \in \mathcal{N}} \pi_{n}^{\exp ^{(r-1)}}=1,
$$




$$
\begin{aligned}
\lambda_{j}^{(r-1)}(l) & =v_{j}^{(r-1)}(l+1) \frac{\pi_{j}^{\exp p^{(r-1)}}(l+1)}{\pi_{j}^{\exp (r-1)}(l)}, \quad l \in\left\{0,1, \ldots, c_{j}-1\right\}, \\
v_{j}^{(r)}(i) & =\lambda_{j}^{(r-1)}(i-1) \frac{\pi_{j}^{(r-1)}(i-1)}{\pi_{j}^{(r-1)}(i)}, \quad i \in\left\{1,2, \ldots, c_{j}\right\}, j \in\{1,2, \ldots, J\},
\end{aligned}
$$

where $\lambda_{j}^{(r)}(l)$ and $v_{j}^{(r)}(i)$ denote the state dependent Poisson arrival rate and throughput of the open QN defined by $\mathcal{J}^{(j)}$ when there are $l$ and $i$ customers at iteration $r$, respectively. Furthermore, $\pi^{\exp (r)}$ is the steady-state vector of the state dependent exponential network constructed by replacing the phase-type service distribution of queue $j$ with the state dependent exponential service time with rate $\mu_{j}^{(r)}(i)=v_{j}^{(r)}(i)$ and $\pi_{j}^{\exp ^{(r)}}(i)$ is the marginal steady-state probability of having $i$ customers in queue $j$ in the exponential network. Finally, $\pi_{j}^{(r)}$ is the steady-state vector of $\mathcal{J}^{(j)}$ and $\pi_{j}^{(r)}(i)$ is the marginal steady-state probability of having $i$ customers in $\mathcal{J}^{(j)}$ at iteration $r$. Observe that the computation of $\lambda_{j}^{(r-1)}$ and $v_{j}^{(r)}$ for $j \in\{1,2, \ldots, J\}$ can be vectorized without difficulty if the elementwise division operator for two vectors of the same length is available.

Each closed QN in Fig. 4 is modeled hierarchically by defining the queue in the subnetwork and the slack queue as two separate LLMs, and constructing the HLM matrix which represents the interactions among the two LLMs. Consequently, the ML method is utilized to compute $\pi_{j}^{(r)}$ for $j \in\{1,2, \ldots, J\}$ in step 4 of Yao and Buzacott's method in Table 2 at iteration $r$.

\section{Numerical results}

The methods of CA, MVABLO, M, YB, ML, and SOR are implemented as part of a software tool in MATLAB (see, for instance, Chapman 2002) using m-files. A total of 67 problems arising from five different closed QNs with phase-type service distributions and arbitrary buffer sizes are analyzed for the utilizations and mean lengths of queues using the software tool. The experiments are performed on a Pentium 3.0 gigahertz with 1 Gigabytes of memory and the methods are compared for their accuracy and efficiency.

The experiments are conducted using two configurations of the ML method. These are $\mathrm{V}$ cycle with fixed aggregation order and $\mathrm{F}$ cycle with circular aggregation order for LLMs (see Buchholz and Dayar 2004 for details). The ML method assumes a stopping tolerance of $10^{-15}$ on the residual 1-norm. The results of the configuration which performs a smaller number of floating-point operations (flops) are reported. The ML method uses SOR with relaxation parameter 1.0, hence, Gauss-Seidel (GS), as the smoother, and performs one preand one post-smoothing at each level in all experiments. A stopping tolerance of $10^{-4}$ is used on the approximate error of performance measures as dictated by the M and YB methods and the maximum number of fixed-point iterations is set to 50. The subnetworks resulting from decomposition in the M and YB methods are solved using the ML method. When computing the steady-state vector of the coarsest generator matrix in the $\mathrm{M}$ method and the steady-state vector of the state dependent exponential network in the YB method, Gaussian elimination is employed if the order of the matrix is less than 500; otherwise, BiCGstab with incomplete LU (ILU) preconditioning (see, for instance, (Saad 2003, Chaps. 7 and 10)) and a drop tolerance of $10^{-5}$ is used. Exact solutions of the problems are obtained via the ML and GS methods. Both methods assume a stopping tolerance of $10^{-15}$ on the residual 1-norm 
and the iteration is stopped prematurely if the desired tolerance is not obtained within 1,000 iterations or 100,000 seconds. Results obtained by approximative methods are compared with results of the ML method and relative errors are provided using the 1-norm.

The problems considered assume three kinds of phase-type service distributions. These are hypoexponential and hyperexponential distributions with two phases and Erlang distribution with five phases. However, this should not be understood to mean that the methods and the software tool are able to work with only these numbers of phases. Now, let the transition rate matrices of a hyperexponential distribution with two phases, a hypoexponential distribution with two phases, and an Erlang distribution with five phases be represented respectively as

$$
T_{\text {Hyper }}^{(i)}=\left(\begin{array}{cc}
-\delta_{1}^{(i)} & 0 \\
0 & -\delta_{2}^{(i)}
\end{array}\right), \quad T_{\text {Hypo }}^{(j)}=\left(\begin{array}{cc}
-\gamma_{1}^{(j)} & \gamma_{1}^{(j)} \\
0 & -\gamma_{2}^{(j)}
\end{array}\right)
$$

and

$$
T_{\text {Erlang }}^{(k)}=\left(\begin{array}{ccccc}
-\zeta^{(k)} & \zeta^{(k)} & 0 & 0 & 0 \\
0 & -\zeta^{(k)} & \zeta^{(k)} & 0 & 0 \\
0 & 0 & -\zeta^{(k)} & \zeta^{(k)} & 0 \\
0 & 0 & 0 & -\zeta^{(k)} & \zeta^{(k)} \\
0 & 0 & 0 & 0 & -\zeta^{(k)}
\end{array}\right)
$$

for some $i, j, k \in\{1,2, \ldots, J\}$. Then $\operatorname{Hyper}^{(i)}\left(\delta_{1}^{(i)}, \delta_{2}^{(i)}, \alpha^{(i)}\right), \operatorname{Hypo}^{(j)}\left(\gamma_{1}^{(j)}, \gamma_{2}^{(j)}\right)$, and Erlang ${ }^{(k)}\left(\zeta^{(k)}, t_{k}\right)$ denote the hyperexponential and hypoexponential distributions with 2 phases and the Erlang distribution with $t_{k}=5$ phases as described, respectively. The initial distribution vectors $\alpha^{(j)}$ and $\alpha^{(k)}$ are not specified for hypoexponential and Erlang distributions since they are of the form $(1,0, \ldots, 0)$ with appropriate length.

The two other parameters for a closed QN are the number of customers in the network and buffer sizes of queues. The number of customers in the network is defined by $K$ and the buffer sizes of queues are defined by the vector $b$ such that $b_{j}$ denotes the buffer size of queue $j$ for $j=\{1,2, \ldots, J\}$. All parameters appear in the captions of Figs. 5 through 9, which depict the topologies of problems 1 through 5. Each problem is further defined by its routing probabilities among queues, which are indicated in the figure depicting the closed QN's topology. The problems have 3, 6, and 8 queues. Problem 5 for $K=9$ has more than one million states. Since buffer sizes of queues in each closed QN are finite, we may have different subnetwork topologies in method $\mathrm{M}$ for different numbers of customers.

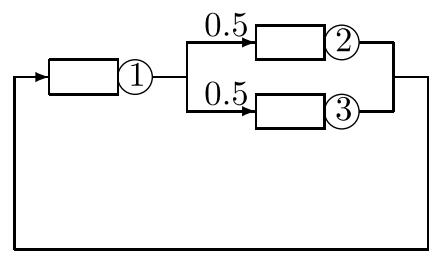

Fig. 5 Problem 1 (Marie's first example). $K=6 ; b=(6,6,6)$; Original: $\operatorname{Hyper}^{(1)}(1.990049503712809$, $9.950496287190580 \mathrm{e}-3, \quad(9.950247518564047 \mathrm{e}-1, \quad 4.975248143595290 \mathrm{e}-3)), \quad \operatorname{Erlang}^{(2)}(1.0,2)$, Erlang ${ }^{(3)} \quad(2.0,2) ; \quad$ Balanced: Hyper $^{(1)} \quad(1.990049503712809, \quad 9.950496287190580 \mathrm{e}-3$, (9.950247518564047e-1, 4.975248143595290e-3)), $\operatorname{Erlang}^{(2)}(1.0,2), \operatorname{Erlang}^{(3)}(1.0,2)$; Unbalanced: Hyper $^{(1)} \quad(1.990049503712809, \quad 9.950496287190580 \mathrm{e}-3, \quad(9.950247518564047 \mathrm{e}-1$, 4.975248143595290e-3)), Erlang ${ }^{(2)}(10.0,2), \operatorname{Erlang}^{(3)}(0.1,2)$ 


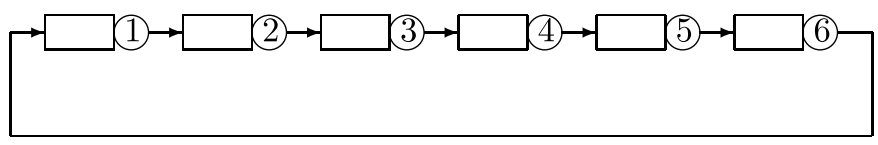

Fig. 6 Problem 2. $K \in\{5,6,7,8\} ; b \in\{(8,5,8,6,8,7),(8,4,8,4,8,4)\}$; Balanced: Hypo $^{(1)}(3.0,1.5)$, Erlang ${ }^{(2)}(5.0,5), \mathrm{Hypo}^{(3)}(1.2,4.0), \mathrm{Hyper}^{(4)}(1.0,1.0,(0.25,0.75))$, Erlang ${ }^{(5)}(5.0,5)$, Hyper ${ }^{(6)}$

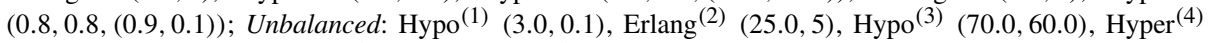
$(0.7,0.7,(0.25,0.75))$, Erlang ${ }^{(5)}(0.1,5)$, Hyper $^{(6)}(0.001,0.001,(0.9,0.1))$

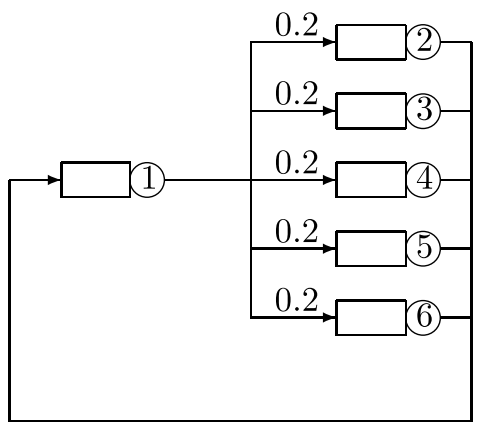

Fig. 7 Problem 3. $K \in\{5,6,7,8\} ; b \in\{(8,5,7,8,8,6),(8,4,4,8,8,4)\}$; Balanced: Hypo ${ }^{(1)}(3.0,1.5)$, Erlang ${ }^{(2)}(1.0,5), \mathrm{Hypo}^{(3)}(0.2,2.0), \mathrm{Hyper}^{(4)}(0.2,0.2,(0.25,0.75))$, Erlang ${ }^{(5)}(1.0,5)$, Hyper ${ }^{(6)}$ $(0.2,0.2,(0.9,0.1))$; Unbalanced: Hypo ${ }^{(1)}(3.0,0.1)$, Erlang ${ }^{(2)}(25.0,5), \mathrm{Hypo}^{(3)}(70.0,60.0)$, Hyper ${ }^{(4)}$ $(0.7,0.7,(0.25,0.75))$, Erlang ${ }^{(5)}(0.1,5)$, Hyper $^{(6)}(0.001,0.001,(0.9,0.1))$

In relation to this, we considered balanced and unbalanced service demands in the subnetworks. The decompositions resulting in method $\mathrm{M}$ for problems 1 through 5 and the results of experiments for 67 problems are reported in (Meriç 2007a, Chap. 6).

The results of the experiments can be summarized as follows. CA and MVABLO produce acceptable results for problems which have queues with balanced service demands and relatively small number of queues subject to blocking. On the other hand, M and YB provide relatively more accurate results for all problems, and they yield results with at least two digits accuracy for unbalanced cases. Also, unlike the results obtained with CA and MVABLO, an increase in the number of queues subject to blocking causes little or no effect in the results obtained with $\mathrm{M}$ and $\mathrm{YB}$. Therefore, $\mathrm{M}$ and $\mathrm{YB}$ arise as better methods than $\mathrm{CA}$ and MVABLO for analyzing problems with unbalanced service demands and a relatively large number of queues subject to blocking. When we compare the accuracies of M and YB, especially in the problems with unbalanced service demands and a relatively large number of queues subject to blocking, we see that $\mathrm{M}$ can produce at least half a digit more accurate results for utilization values than $\mathrm{YB}$. When we compare the efficiencies of $\mathrm{M}$ and $\mathrm{YB}$, we see that the number of flops performed by YB to compute arrival rates of queues mostly depends on the number of flops performed for obtaining the solution of the exponential network generated at each iteration. Therefore, for problems which require a relatively small number of flops for the solution of the exponential network, YB executes less flops than M. Also, for problems which result in subnetworks with a relatively large number of queues for M, YB may end up performing less flops than $M$ through its fixed-point approximation process. Consequently, efficiencies of $\mathrm{M}$ and $\mathrm{YB}$ depend heavily on the particular problem. When we compare ML and GS, we see that ML achieves convergence within 100 iterations 


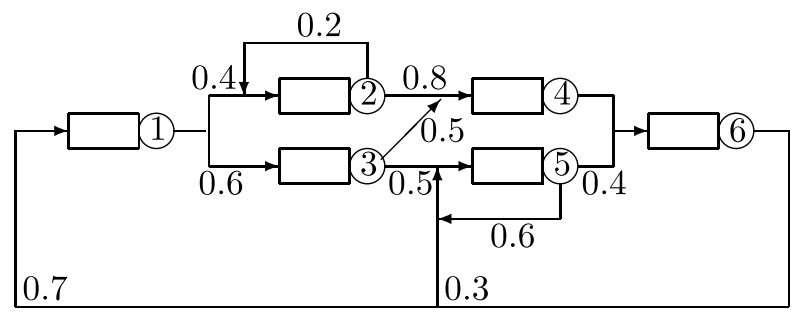

Fig. 8 Problem 4. $K \in\{5,6,7,8\} ; b \in\{(8,5,7,8,8,6),(8,4,4,8,8,4)\}$; Balanced: Hypo ${ }^{(1)}(3.0,1.5)$, Erlang ${ }^{(2)}(2.5,5)$, Hypo $^{(3)}(0.8,2)$, Hyper $^{(4)}(0.7,0.7,(0.25,0.75))$, Erlang ${ }^{(5)}(9.0,5)$, Hyper $(6)$ $(1.4,1.4,(0.9,0.1))$; Unbalanced: $\mathrm{Hypo}^{(1)}(3.0,0.1)$, $\operatorname{Erlang}^{(2)}(25.0,5)$, $\mathrm{Hypo}^{(3)}(70.0,60.0)$, Hyper $^{(4)}(0.7,0.7,(0.25,0.75))$, Erlang $^{(5)}(0.1,5)$, Hyper $^{(6)}(0.001,0.001,(0.9,0.1))$

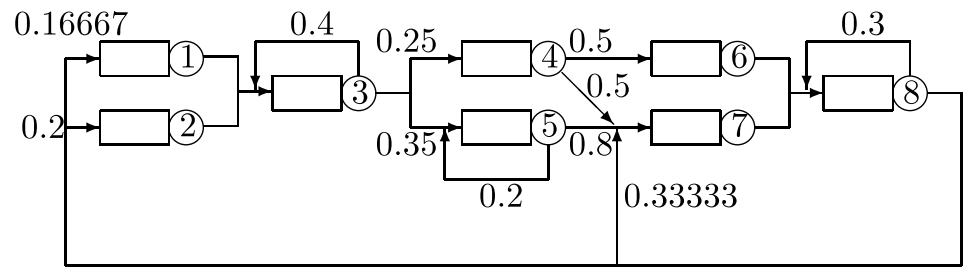

Fig. 9 Problem 5. $K \in\{6,7,8,9\} ; \quad b \in\{(8,9,9,6,6,9,9,7), \quad(5,9,9,5,5,9,9,5)\} ;$ Balanced: Hypo $^{(1)}(1.5,2.0)$, Hyper $^{(2)}(1.1,1.1,(0.1,0.9))$,Erlang $^{(3)}(18.0,5)$ Hyper $^{(4)}(0.9,0.9,(0.85,0.15))$, Hypo $^{(5)}(1.5,10.0), \operatorname{Hypo}^{(6)}(0.5,4.0), \operatorname{Hyper}^{(7)}(3.5,3.5,(0.25,0.75)), \quad \operatorname{Erlang}^{(8)}(30,5)$; Unbalanced: Hypo ${ }^{(1)}(200.0,90.0)$, Hyper $^{(2)}(1,000.0,1,000.0,(0.1,0.9))$, Erlang ${ }^{(3)}(0.15,5)$, Hyper ${ }^{(4)}$ $(0.008,0.008,(0.85,0.15))$, Hypo $^{(5)}(8,000.0,5,000.0)$, Hypo $^{(6)}(0.1,0.05)$, Hyper ${ }^{(7)}(10.0,10.0$, $(0.25,0.75))$, Erlang $^{(8)}(30.0,5)$

in all problems. Yet, GS does not converge within 1,000 iterations or 100,000 seconds for some of the problems. Clearly, the number of iterations determines the number of flops performed by the methods, and ML performs less flops than GS in almost all problems. Even though GS takes less space in memory than ML, in most of the problems ML requires less memory than the sparse representation of the generator matrix, thereby, being capable of solving variants of the problems with relatively large numbers of customers. Since $\mathrm{M}$ and YB are based on decomposition, the space requirements of $\mathrm{M}$ and $\mathrm{YB}$ are smaller than that of ML and GS for relatively large problems. Indeed, it is verified that the usage of ML in $\mathrm{M}$ and $\mathrm{YB}$ introduces another dimension of scalability to the space requirements of the two methods.

These results are summarized in Table 3. The table consists of six rows and eight columns. Rows of the table correspond to the methods. Its columns respectively correspond to average number of iterations performed by the methods (oIter), average number of iterations performed by ML per one iteration of $\mathrm{M}$ and $\mathrm{YB}$ or average number of smoothings at the finest level for ML (iIter), average time taken by the methods in seconds (T), average number of megaflops (MF) performed by the methods, average memory requirement of the methods in megabytes (MB), average relative error in the utilization of queues $(\operatorname{RE}(\rho))$, average relative error in the mean queue lengths $(\mathrm{RE}(\mathrm{E}[\mathrm{X}]))$, and average 1-norm of the residual vectors for ML and GS. Timing results of the methods are given for demonstration purposes only. Indeed, experiments in MATLAB should not be expected to run in times that 
Table 3 Average values for 67 problems

\begin{tabular}{lclrrrrrr}
\hline Method & oIter & iIter & \multicolumn{1}{l}{$\mathrm{T}$} & $\mathrm{MF}$ & $\mathrm{MB}$ & $\mathrm{RE}(\rho)$ & $\mathrm{RE}(\mathrm{E}[\mathrm{X}])$ & $\mathrm{Res}$ \\
\hline CA & $\mathrm{n} / \mathrm{a}$ & $\mathrm{n} / \mathrm{a}$ & 0 & 0 & 0.0 & $2 \mathrm{e}-1$ & $2 \mathrm{e}-1$ & $\mathrm{n} / \mathrm{a}$ \\
MVABLO & $\mathrm{n} / \mathrm{a}$ & $\mathrm{n} / \mathrm{a}$ & 0 & 0 & 0.0 & $2 \mathrm{e}-1$ & $2 \mathrm{e}-1$ & $\mathrm{n} / \mathrm{a}$ \\
M & 4 & 7 & 375 & 202 & 0.3 & $6 \mathrm{e}-3$ & $8 \mathrm{e}-3$ & $\mathrm{n} / \mathrm{a}$ \\
YB & 5 & 5 & 90 & 5,204 & 0.4 & $9 \mathrm{e}-3$ & $2 \mathrm{e}-2$ & $\mathrm{n} / \mathrm{a}$ \\
ML & 23 & 2 & 8,863 & 14,550 & 11.1 & $\mathrm{n} / \mathrm{a}$ & $\mathrm{n} / \mathrm{a}$ & $4 \mathrm{e}-16$ \\
GS & 635 & $\mathrm{n} / \mathrm{a}$ & 27,506 & 13,213 & 3.9 & $5 \mathrm{e}-4$ & $2 \mathrm{e}-3$ & $5 \mathrm{e}-5$ \\
\hline
\end{tabular}

Table 4 Flop and space requirements of M and YB methods' Kronecker and sparse implementations

\begin{tabular}{|c|c|c|c|c|c|}
\hline \multirow[t]{2}{*}{ Problem set } & \multirow[t]{2}{*}{ Method } & \multicolumn{2}{|c|}{ Kronecker } & \multicolumn{2}{|l|}{ Sparse } \\
\hline & & MF & MB & MF & MB \\
\hline \multirow[t]{2}{*}{1} & M & 4 & 0.0 & 0 & 0.0 \\
\hline & YB & 4 & 0.0 & 0 & 0.0 \\
\hline \multirow[t]{2}{*}{2} & M & 6 & 0.0 & 2 & 0.1 \\
\hline & YB & 132 & 0.1 & 203 & 0.1 \\
\hline \multirow[t]{2}{*}{3} & $\mathrm{M}$ & 202 & 0.3 & 34,903 & 0.5 \\
\hline & YB & 187 & 0.2 & 238 & 0.2 \\
\hline \multirow[t]{2}{*}{4} & M & 77 & 0.1 & 880 & 0.2 \\
\hline & YB & 313 & 0.1 & 2,222 & 0.2 \\
\hline \multirow[t]{2}{*}{5} & $\mathrm{M}$ & 623 & 0.5 & 127,442 & 1.0 \\
\hline & YB & 13,154 & 0.9 & 51,382 & 0.9 \\
\hline
\end{tabular}

are consistent with flop count analyses. One can find a detailed explanation of this situation in Genz et al. (1991).

In order to see the merits of the proposed Kronecker implementations with respect to sparse implementations, we provide Table 4. In this group of experiments, we have set the Kronecker based ML solver's parameters of cycle type to $\mathrm{V}$ and the order of aggregation to fixed for consistency. We remark that these two parameter values correspond to the simplest forms of the Kronecker based implementations. All other parameters remain as in the previous group of experiments. Sparse implementations of the M and YB methods are accomplished by generating the matrices corresponding to the respective subnetworks onthe-fly and then solving for their steady-state vectors using the built-in Gaussian elimination method of Matlab at each iteration. The flop counts and space requirements are averaged in each problem set, which respectively have $3,16,16,16,16$ problems. There are three problems which could not be solved; all appear in problem set 5 for $K=9$ (that is, the largest problems) and correspond to the sparse implementation of the YB method with balanced service demands for both buffer size vectors, and with unbalanced service demands for $b=(5,9,9,5,5,9,9,5)$. In order to make a fair comparison, we have excluded the results of the same three problems obtained with the Kronecker implementation of the YB method. Hence, for problem set 5, the averaging for the YB method is done over 13 problems, and the results exclude those belonging to the three of the four largest problems.

Now, we mention some general observations. It is not worthwhile to use Kronecker implementations of the $\mathrm{M}$ and $\mathrm{YB}$ methods in problem set 1 . This is an expected result since in 
this case the resulting problems are very small. However, starting from problem set 2 with the YB method, Kronecker implementations start paying off with respect to sparse implementations, always with smaller flop counts and sometimes with smaller space requirements. The situation regarding the space requirements of sparse implementations is understandable since the input matrices that correspond to the subnetworks are never very large, are stored in sparse format, and are solved using sparse Gaussian elimination. Hence, this does not generate overly large fill-in compared to the existing space requirements of the Kronecker implementations. Furthermore, the gain in the YB method with the Kronecker implementation is never as significant as that obtained with the $\mathrm{M}$ method since the subnetworks from which the sparse matrices are generated have only one queue with the YB method. This result is also in line with the expectations. With regards to specific observations associated with the $\mathrm{M}$ and $\mathrm{YB}$ methods, it is problem set 3 which has the largest number of queues in a subnetwork for the M method (4 queues for $K=8$ when $b=(8,5,7,8,8,6)$ and 4 queues for all $K$ values when $b=(8,4,4,8,8,4))$. The results indicate that the Kronecker implementation of the $\mathrm{M}$ method benefits considerably from such a situation. A similar result also holds for problem set 5 .

\section{Conclusion}

In this paper, we considered two approximative iterative methods based on decomposition from the literature, namely Marie's method and Yao and Buzacott's method. It is shown that these methods can be used for analyzing relatively large closed queueing networks with phase-type service distributions and arbitrary buffer sizes. While analyzing such closed queueing networks, subnetworks resulting from decomposition are represented using Kronecker products. This is shown to add another level of scalability to the methods by requiring less space than the ordinary sparse representation of subnetworks. Furthermore, the Kronecker representation of subnetworks enables the use of a multilevel method in their solution.

The effect of using the multilevel method is analyzed through a set of numerical experiments, which show that the number of iterations and floating-point operations taken by the multilevel method are generally much smaller than those of the Gauss-Seidel method. Thus, the employment of the multilevel method within Marie's method and Yao and Buzacott's method makes the two methods more efficient. A comparison of the Kronecker implementations with sparse implementations of these two methods reinforces this result further. The methods are also compared with two analytical methods from the literature, namely the convolution algorithm and Akyildiz's mean value analysis, and the cases in which Marie's method and Yao and Buzacott's method yield better approximations are identified. Indeed, Marie's method and Yao and Buzacott's method yield results with relative errors less than $10^{-5}$ for unbalanced cases of problems 2, 3, and 4. We also see that Marie's method and Yao and Buzacott's method yield more accurate results for relatively crowded closed QNs with unbalanced service demands. In general, Marie's method approximates the performance measures of utilization and average number of customers in queues at least half a digit better than Yao and Buzacott's method. The efficiency of the two methods may present different behavior for different kinds of networks. For instance, an unbalanced decomposition of the network in Marie's method may cause Marie's method to be less efficient than Yao and Buzacott's method, whereas Yao and Buzacott's method may be less efficient than Marie's method for problems which have a relatively large high level model tieing together 
the low level models in the Kronecker representation of the closed QN or which the steadystate solution of the state dependent exponential network requires an extensive number of floating-point operations to be computed in sparse representation.

\section{References}

Akyildiz, I. F. (1988a). On the exact and approximate throughput analysis of closed queueing networks with blocking. IEEE Transactions on Software Engineering, 14, 62-71.

Akyildiz, I. F. (1988b). Mean value analysis for blocking queueing networks. IEEE Transactions on Software Engineering, 14, 418-428.

Altiok, T. (1989). Approximate analysis of queues in series with phase-type service time and blocking. $O p$ erations Research, 37, 601-610.

Baskett, F., Chandy, K. M., Muntz, R. R., \& Palacios, F. G. (1975). Open, closed, and mixed networks of queues with different classes of customers. Journal of the ACM, 22, 248-260.

Buchholz, P. (1994). A class of hierarchical queueing networks and their analysis. Queueing Systems, 15, 59-80.

Buchholz, P., \& Dayar, T. (2004). Comparison of multilevel methods for Kronecker-based Markovian representations. Computing, 73, 349-371.

Buchholz, P., \& Dayar, T. (2007). On the convergence of a class of multilevel methods for large, sparse Markov chains. SIAM Journal on Matrix Analysis and Applications, 29, 1025-1049.

Buzen, J. P. (1973). Computational algorithms for closed queueing networks with exponential servers. Communications of the ACM, 16, 527-531.

Chapman, S. J. (2002). MATLAB programming for engineers. Pacific Grove: Brooks/Cole Publishing.

Davio, M. (1981). Kronecker products and shuffle algebra. IEEE Transactions on Computers, 30, 116-125.

Dayar, T. (2006). Analyzing Markov chains based on Kronecker products. In A. N. Langville \& W. J. Stewart (Eds.), MAM 2006: Markov anniversary meeting (pp. 279-300). Raleigh: Boson Books.

Frein, Y., \& Dallery, Y. (1989). Analysis of cyclic queueing networks with finite buffers and blocking before service. Performance Evaluation, 10, 197-210.

Fernandes, P., Plateau, B., \& Stewart, W. J. (1998). Efficient descriptor-vector multiplications in stochastic automata networks. Journal of the ACM, 45, 381-414.

Genz, A., Lin, Z., Jones, C., Luo, D., \& Prenzel, T. (1991). Fast givens goes slow in MATLAB. ACM SIGNUM Newsletter, 26, 11-16.

Gordon, W. J., \& Newell, G. F. (1967). Closed queueing systems with exponential servers. Operations Research, 15, 252-267.

Mainkar, V., \& Trivedi, K. S. (1996). Sufficient conditions for existence of a fixed point in stochastic reward net-based iterative models. IEEE Transactions on Software Engineering, 22, 640-653.

Marie, R. A. (1979). An approximate analytical method for general queueing networks. IEEE Transactions on Software Engineering, 5, 530-538.

Meriç, A. (2007a). Kronecker representation and decompositional analysis of closed queueing networks with phase-type service distributions and arbitrary buffer sizes. M.S. Thesis, Department of Computer Engineering, Bilkent University, Ankara, Turkey. Available from http://www.cs.bilkent.edu.tr/tech-reports/2007/BU-CE-0705.pdf.

Meriç, A. (2007b). Software for Kronecker representation and decompositional analysis of closed queueing networks with phase-type service distributions and arbitrary buffer sizes. Available from http://www.cs.bilkent.edu.tr/ tugrul/software.html.

Perros, H. G., Nilsson, A. A., \& Liu, Y.-C. (1988). Approximate analysis of product-form type queueing networks with blocking and deadlock. Performance Evaluation, 8, 19-39.

Reiser, M., Lavenberg, S. S. (1980). Mean value analysis of closed multichain queueing networks. Journal of the ACM, 22, 313-322.

Saad, Y. (2003). Iterative methods for sparse linear systems. Philadelphia: SIAM Press.

Stewart, W. J. (1994). Introduction to the numerical solution of Markov chains. Princeton: Princeton University Press.

Suri, R., \& Diehl, G. W. (1986). A variable buffer size model and its use in analytical closed queueing networks with blocking. Management Science, 32, 206-225.

Uysal, E., \& Dayar, T. (1998). Iterative methods based on splittings for stochastic automata networks. European Journal of Operational Research, 110, 166-186.

Yao, D. D., \& Buzacott, J. A. (1986). The exponentialization approach to flexible manufacturing systems models with general processing times. European Journal of Operational Research, 24, 410-416. 